International Journal of Modern Physics E (C) World Scientific Publishing Company

\title{
TRANSITION DENSITY AND PRESSURE AT THE INNER EDGE OF NEUTRON STAR CRUSTS
}

\author{
JUN XU and CHE MING KO \\ Cyclotron Institute and Department of Physics and Astronomy, Texas A\&M University, College \\ Station, TX 77843-3366, USA \\ xujun@comp.tamu.edu and ko@comp.tamu.edu \\ LIE-WEN CHEN \\ Department of Physics, Shanghai Jiao Tong University, Shanghai 200240, China \\ Center of Theoretical Nuclear Physics, National Laboratory of Heavy-Ion Accelerator, Lanzhou, \\ 730000, China \\ lwchen@sjtu.edu.cn \\ BAO-AN LI \\ Department of Physics and Astronomy, Texas A\&SM University-Commerce, Commerce, TX \\ 75429-3011, USA \\ Bao-An_Li@tamu-commerce.edu \\ HONG-RU MA \\ Department of Physics, Shanghai Jiao Tong University, Shanghai 200240, China \\ hrma@sjtu.edu.cn \\ Received (received date) \\ Revised (revised date)
}

\begin{abstract}
Using the nuclear symmetry energy that has been recently constrained by the isospin diffusion data in intermediate-energy heavy ion collisions, we have studied the transition density and pressure at the inner edge of neutron star crusts, and they are found to be $0.040 \mathrm{fm}^{-3} \leq \rho_{t} \leq 0.065 \mathrm{fm}^{-3}$ and $0.01 \mathrm{MeV} / \mathrm{fm}^{3} \leq P_{t} \leq 0.26 \mathrm{MeV} / \mathrm{fm}^{3}$, respectively, in both the dynamical and thermodynamical approaches. We have also found that the widely used parabolic approximation to the equation of state of asymmetric nuclear matter gives significantly higher values of core-crust transition density and pressure, especially for stiff symmetry energies. With these newly determined transition density and pressure, we have obtained an improved relation between the mass and radius of neutron stars.
\end{abstract}

\section{Introduction}

Studying the properties of neutron stars, which are among the most mysterious objects in the universe, allows us to test our knowledge of matter under extreme conditions. Theoretical studies have shown that neutron stars are expected to have a liquid core surrounded by a solid inner crust 1 , which extends outward to the 
neutron drip-out region. While the neutron drip-out density $\rho_{\text {out }}$ is relatively well determined to be about $4 \times 10^{11} \mathrm{~g} / \mathrm{cm}^{3}$ or $0.00024 \mathrm{fm}^{-3}$ [2] the transition density $\rho_{t}$ at the inner edge is still largely uncertain mainly because of our very limited knowledge on the nuclear equation of state (EOS), especially the density dependence of the symmetry energy $\left(E_{\mathrm{sym}}(\rho)\right)$ of neutron-rich nuclear matter $3 \mid 4$. These uncertainties have hampered our understanding of many important properties of neutron stars $3|4| 5$ and related astrophysical observations $3|4| 6|7| 8|9| 10|11| 12|13| 14$. Recently, significant progress has been made in constraining the EOS of neutron-rich nuclear matter using terrestrial laboratory experiments (See Ref. 15 for the most recent review). In particular, the analysis of the isospin-diffusion data $16[17 \mid 18$ in heavy-ion collisions has constrained tightly the $E_{\text {sym }}(\rho)$ in exactly the same subsaturation density region around the expected inner edge of neutron star crust. The extracted slope parameter $L=\left.3 \rho_{0} \frac{\partial E_{\mathrm{sym}}(\rho)}{\partial \rho}\right|_{\rho=\rho_{0}}$ in the density dependence of the nuclear symmetry energy was found to be $86 \pm 25 \mathrm{MeV}$, which has further been confirmed by a more recent analysis 19 . With this constrained nuclear symmetry energy, we have obtained an improved determination of the values for the transition density and pressure at the inner edge of neutron star crusts. This has led us to obtain significantly different values for the radius of the Vela pulsar from those estimated previously. Also, we have found that the widely used parabolic approximation (PA) to the EOS of asymmetric nuclear matter gives much larger values for the transition density and pressure, especially for stiff symmetry energies.

\section{Dynamical and Thermodynamical approaches to the stability of npe matter}

The transition density at the inner edge of neutron star crusts can be determined using the dynamical $6|7| 8|9| 20|21| 22$ and the thermodynamical approaches $423 \mid 24$ as well as the approach based on the random phase approximation (RPA) 25126 . Our study was based on the dynamical and thermodynamical approaches.

In the dynamical approach, the stability condition for a homogeneous npe matter against small periodic density perturbations can be well approximated by $6 / 7 / 8 / 9 \mid 21$

$$
V_{\mathrm{dyn}}(k)=V_{0}+\beta k^{2}+\frac{4 \pi e^{2}}{k^{2}+k_{T F}^{2}}>0,
$$

where $k$ is the wavevector of the spatially periodic density perturbations and

$$
\begin{aligned}
V_{0} & =\frac{\partial \mu_{p}}{\partial \rho_{p}}-\frac{\left(\partial \mu_{n} / \partial \rho_{p}\right)^{2}}{\partial \mu_{n} / \partial \rho_{n}}, k_{T F}^{2}=\frac{4 \pi e^{2}}{\partial \mu_{e} / \partial \rho_{e}}, \\
\beta & =D_{p p}+2 D_{n p} \zeta+D_{n n} \zeta^{2}, \quad \zeta=-\frac{\partial \mu_{n} / \partial \rho_{p}}{\partial \mu_{n} / \partial \rho_{n}},
\end{aligned}
$$

with $\mu_{i}$ being the chemical potential of particle type $i$. The three terms in Eq. (1) represent, respectively, the contributions from the bulk nuclear matter, the densitygradient (surface) terms, and the Coulomb interaction. For the coefficients of density-gradient terms, we use the empirical values of $D_{p p}=D_{n n}=D_{n p}=132$ 
$\mathrm{MeV} \cdot \mathrm{fm}^{5}$, which are consistent with those from the Skyrme-Hartree-Fock calculations 21127. At $k_{\min }=\left[\left(\frac{4 \pi e^{2}}{\beta}\right)^{1 / 2}-k_{T F}^{2}\right]^{1 / 2}, V_{\mathrm{dyn}}(k)$ has the minimal value of $V_{\text {dyn }}\left(k_{\min }\right)=V_{0}+2\left(4 \pi e^{2} \beta\right)^{1 / 2}-\beta k_{T F}^{2}$ 6/7/8|9|21, and $\rho_{t}$ is then determined from $V_{\text {dyn }}\left(k_{\min }\right)=0$.

In the thermodynamical approach, the stability conditions for the npe matter are $4 \mid 23228$

$$
-\left(\frac{\partial P}{\partial v}\right)_{\mu}>0, \quad \text { and } \quad-\left(\frac{\partial \mu}{\partial q_{c}}\right)_{v}>0
$$

In the above, $P=P_{b}+P_{e}$ is the total pressure of the npe matter with $P_{b}$ and $P_{e}$ being the contributions from baryons and electrons, respectively; $v$ and $q_{c}$ are the volume and charge per baryon number; and $\mu=\mu_{n}-\mu_{p}$ is the chemical potential of the npe matter. It can be shown that the first inequality in Eq. (2) is equivalent to requiring a positive bulk term $V_{0}$ in Eq. (1) 27, while the second inequality in Eq. (2) is almost always satisfied. Therefore, the thermodynamical stability conditions are simply the limit of the dynamical one for $k \rightarrow 0$ when the surface terms and the Coulomb interaction are neglected.

\section{Results for the transition density and pressure}

We have used in our study a momentum-dependent MDI interaction that is based on the modified finite-range Gogny effective interaction 29. This interaction, which has been extensively studied in our previous work 15 , is exactly the one used in analyzing the isospin diffusion data from heavy-ion reactions $17 / 18$.

Shown in Fig. 1 is the transition density $\rho_{t}$ as a function of the slope parameter $L$ of the symmetry energy from the MDI interaction. For comparisons, we have included results from both the dynamical and thermodynamical approaches with the full EOS and its parabolic approximation, i.e., $E(\rho, \delta)=E(\rho, \delta=$ $0)+E_{\text {sym }}(\rho) \delta^{2}+O\left(\delta^{4}\right)$, where $\delta=\left(\rho_{n}-\rho_{p}\right) / \rho$ is the isospin asymmetry, from the same MDI interaction. With the full MDI EOS, $\rho_{t}$ is seen to decrease almost linearly with increasing $L$ in both approaches consistent with the results obtained in RPA 25. Both dynamical and thermodynamical approaches give very similar results with the former having slightly smaller $\rho_{t}$ than the later (the difference is actually less than $0.01 \mathrm{fm}^{-3}$ ), and this is due to the fact that the density-gradient and Coulomb terms in the dynamical approach make the system more stable and thus lower the transition density. The small difference between the two approaches implies that the effects of density-gradient terms and Coulomb term are, however, unimportant in determining $\rho_{t}$. On the other hand, significantly larger transition densities are obtained in the parabolic approximation, including the predictions by Kubis using the MDI EOS in the thermodynamical approach 23 , especially for stiffer symmetry energies (larger $L$ values). The large error introduced by the parabolic approach is understandable since the $\beta$-stable npe matter is usually highly neutronrich and the contribution from the higher-order terms in $\delta$ is thus appreciable. This 
Jun $X u$ et al.

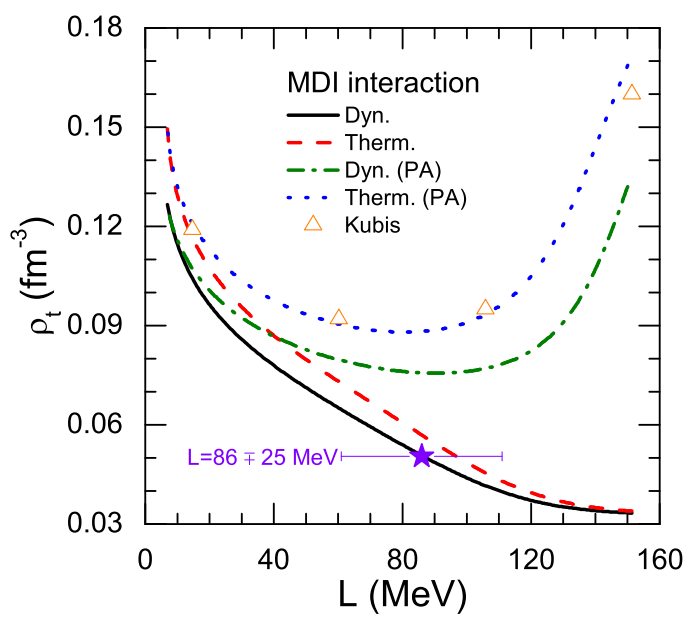

Fig. 1. (Color online) The transition density $\rho_{t}$ as a function of the slope parameter $L$ of the nuclear symmetry energy from the dynamical and thermodynamical approaches with and without the parabolic approximation in the MDI interaction. Taken from Ref. 27.

is especially the case for the stiffer symmetry energy which generally leads to a more neutron-rich npe matter at subsaturation densities. Furthermore, because of the energy curvatures involved in the stability conditions, larger factors are multiplied to the contributions from higher-order terms in the EOS than that multiplied to the quadratic term. These features agree with the early finding 30 that the transition density $\rho_{t}$ is very sensitive to the fine details of the nuclear EOS. According to results from the more complete and realistic dynamical approach, the constrained $L$ limits the transition density to $0.040 \mathrm{fm}^{-3} \leq \rho_{t} \leq 0.065 \mathrm{fm}^{-3}$ as shown in Fig. 1

The transition pressure $P_{t}$ at the inner edge of the neutron star crust is also an important quantity that might be measurable indirectly from observations of pulsar glitches 411. Shown in Fig. 2 is the $P_{t}$ as a function of $L$ and $\rho_{t}$ by using the dynamical approach with both the full MDI EOS and its PA. Again, it is seen that the PA leads to huge errors for large (small) $L\left(\rho_{t}\right)$ values. For the full MDI EOS, the $P_{t}$ decreases (increases) with increasing $L\left(\rho_{t}\right)$ while it displays a complex relation with $L$ or $\rho_{t}$ in PA. From the constrained $L$ values, the value of $P_{t}$ is limited between $0.01 \mathrm{MeV} / \mathrm{fm}^{3}$ and $0.26 \mathrm{MeV} / \mathrm{fm}^{3}$.

\section{Improved constraint on the radius-mass relation of neutron stars}

The constrained values of $\rho_{t}$ and $P_{t}$ have important implications in many properties of neutron stars $4|8| 12 \mid 21$. As an example, we have examined their effect on constraining the mass-radius $(M-R)$ correlation of neutron stars. The crustal fraction 


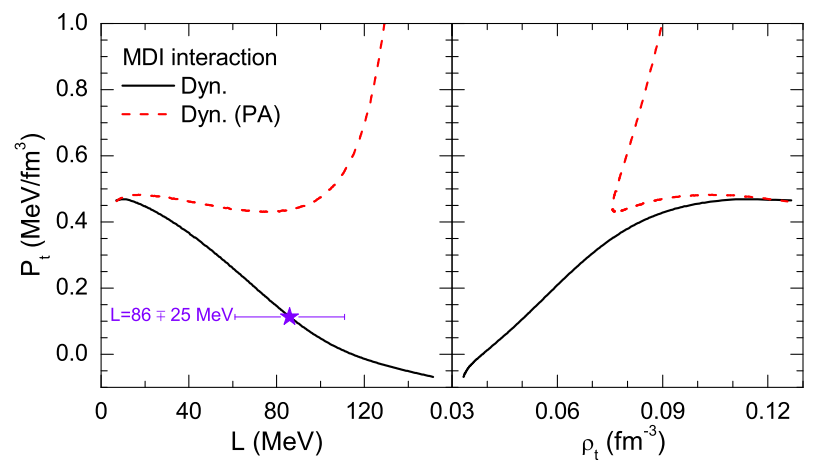

Fig. 2. (Color online) The transition pressure $P_{t}$ as a functionof $L$ and $\rho_{t}$ by using the dynamical approach with and without the parabolic approximation in the MDI interaction. Taken from Ref. 27 .

of the total moment of inertia $\Delta I / I$ of a neutron star can be well approximated by $3|4| 11$

$$
\frac{\Delta I}{I} \approx \frac{28 \pi P_{t} R^{3}}{3 M c^{2}} \frac{\left(1-1.67 \xi-0.6 \xi^{2}\right)}{\xi}\left[1+\frac{2 P_{t}\left(1+5 \xi-14 \xi^{2}\right)}{\rho_{t} m_{b} c^{2} \xi^{2}}\right]^{-1},
$$

where $m_{b}$ is the baryon mass and $\xi=G M / R c^{2}$ with $G$ being the gravitational constant. As stressed in Ref. $\frac{3}{,}, \Delta I / I$ depends sensitively on the symmetry energy at subsaturation densities through $P_{t}$ and $\rho_{t}$, but there is no explicit dependence on the higher-density EOS. So far, the only known limit of $\Delta I / I>0.014$ was extracted from studying the glitches of Vela pulsar 11 . This together with the upper bounds on $P_{t}$ and $\rho_{t}\left(\rho_{t}=0.065 \mathrm{fm}^{-3}\right.$ and $\left.P_{t}=0.26 \mathrm{MeV} / \mathrm{fm}^{3}\right)$ sets approximately a minimum radius of $R \geq 4.7+4.0 M / M_{\odot} \mathrm{km}$ for the Vela pulsar. The radius of Vela pulsar is predicted to exceed $10.5 \mathrm{~km}$ should it have a mass of $1.4 M_{\odot}$. We notice that a constraint of $R \geq 3.6+3.9 M / M_{\odot} \mathrm{km}$ for this pulsar has previously been derived in Ref. 11 by using $\rho_{t}=0.075 \mathrm{fm}^{-3}$ and $P_{t}=0.65 \mathrm{MeV} / \mathrm{fm}^{3}$. However, the constraint obtained in our study using for the first time data from both the terrestrial laboratory experiments and astrophysical observations is more stringent.

The above constraints are shown in Fig. 3 together with the $\mathrm{M}-\mathrm{R}$ relation obtained by solving the Tolman-Oppenheimer-Volkoff (TOV) equation. In the latter, we have used the well-known BPS EOS 6 for the outer crust. In the inner crust with $\rho_{\text {out }}<\rho<\rho_{t}$, the EOS is largely uncertain and following Ref. 26 , we use an EOS of the form $P=a+b \epsilon^{4 / 3}$ with the constants $a$ and $b$ determined by the total pressure $P$ and total energy density $\epsilon$ at $\rho_{\text {out }}$ and $\rho_{t}$. The full MDI EOS and its parabolic approximation with $x=0$ and $x=-1$ are used for the uniform liquid core with $\rho \geq \rho_{t}$. Assuming that the core consists of only the npe matter without possible new degrees of freedom or phase transitions at high densities, the PA leads to a larger radius for a fixed mass compared to the full MDI EOS. Furthermore, using 


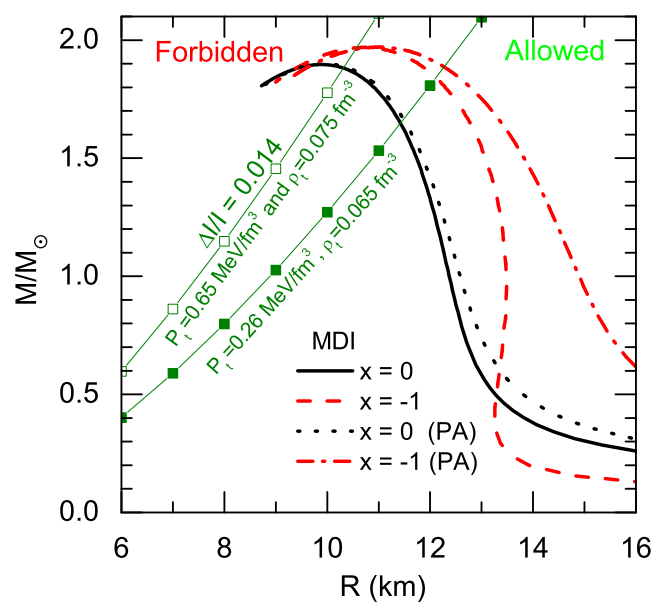

Fig. 3. (Color online) The mass-radius relation $M-R$ of static neutron stars from the full EOS and its parabolic approximation in the MDI interaction with $x=0$ and $x=-1$. For the Vela pulsar, the constraint of $\Delta I / I>0.014$ limits the allowed masses and radii. See text for details. Taken from Ref. 27 with small modifications.

the full MDI EOS with $x=0$ and $x=-1$ constrained by the heavy-ion reaction experiments, the radius of a canonical neutron star of $1.4 M_{\odot}$ is tightly constrained within $11.9 \mathrm{~km}$ to $13.2 \mathrm{~km}$.

\section{Summery}

Using the MDI EOS of neutron-rich nuclear matter constrained by recent isospin diffusion data from heavy-ion reactions in the same sub-saturation density range as the neutron star crust, we have determined the density and pressure at the inner edge, that separates the liquid core from the solid crust of neutron stars, to be 0.040 $\mathrm{fm}^{-3} \leq \rho_{t} \leq 0.065 \mathrm{fm}^{-3}$ and $0.01 \mathrm{MeV} / \mathrm{fm}^{3} \leq P_{t} \leq 0.26 \mathrm{MeV} / \mathrm{fm}^{3}$, respectively. These constraints have allowed us to determine an improved mass-radius relation for neutron stars. Furthermore, we have found that the widely used parabolic approximation to the EOS of asymmetric nuclear matter leads to significantly higher core-crust transition densities and pressures, especially for stiff nuclear symmetry energies.

\section{Acknowledgements}

This work was supported in part by U.S. National Science Foundation under Grant No. PHY-0758115, PHY-0652548 and PHY-0757839, the Welch Foundation under Grant No. A-1358, the Research Corporation under Award No. 7123, the Texas Co- 
ordinating Board of Higher Education Award No. 003565-0004-2007, the National Natural Science Foundation of China under Grant Nos. 10575071 and 10675082, MOE of China under project NCET-05-0392, Shanghai Rising-Star Program under Grant No. 06QA14024, the SRF for ROCS, SEM of China, the National Basic Research Program of China (973 Program) under Contract No. 2007CB815004.

\section{References}

1. N. Chamel and P. Haensel, Living Rev. Relativity, 11 (2008) 10.

2. S. B. Ruster, M. Hempel, and J. Schaffner-Bielich, Phys. Rev. C 73 (2006) 035804.

3. J.M. Lattimer and M. Prakash, Phys. Rep. 333-334 (2000) 121; Astrophys. J. 550 (2001) 426.

4. J.M. Lattimer and M. Prakash, Phys. Rep. 442 (2007) 109.

5. J.M. Lattimer and M. Prakash, Science 304 (2004) 536.

6. G. Baym, C. Pethick and P. Sutherland, Astrophys. J. 170 (1971) 299.

7. G. Baym, H. A. Bethe and C. J. Pethick, Nucl. Phys. A 175 (1971) 225.

8. C. J. Pethick and D. G. Ravenhall, Ann. Rev. Nucl. Part. Sci. 45 (1995) 429.

9. C. J. Pethick, D. G. Ravenhall and C. P. Lorenz, Nucl. Phys. A 584 (1995) 675.

10. A.W. Steiner, M. Prakash, J.M. Lattimer, and P.J. Ellis, Phys. Rep. 410 (2005) 325.

11. B. Link, R. I. Epstein, J.M. Lattimer, Phys. Rev. Lett. 83 (1999) 3362.

12. C.J. Horowitz, M. A. Pérez-García and J. Piekarewicz, Phys. Rev. C 69 (2004) 045804; C.J. Horowitz et al., Phys. Rev. C 70 (2004) 065806.

13. A. Burrows, S. Reddy, and T. A. Thompson, Nucl. Phys. A 777 (2006) 356.

14. B. J. Owen, Phys. Rev. Lett. 95 (2005) 211101.

15. B.A. Li, L.W. Chen, and C.M. Ko, Phys. Rep. 464 (2008) 113.

16. M.B. Tsang et al., Phys. Rev. Lett. 92 (2004) 062701.

17. L.W. Chen, C.M. Ko, and B.A. Li, Phys. Rev. Lett. 94 (2005) 032701.

18. B.A. Li and L.W. Chen, Phys. Rev. C 72 (2005) 064611.

19. M.B. Tsang et al., Phys. Rev. Lett. 102 (2009) 112701.

20. F. Douchin and P. Haensel, Phys. Lett. B 485 (2000) 107.

21. K. Oyamatsu and K. Iida, Phys. Rev. C 75 (2007) 015801.

22. C. Ducoin, Ph. Chomaz and F. Gulminelli, Nucl. Phys. A 789 (2007) 403.

23. S. Kubis, Phys. Rev. C 76 (2007) 035801; Phys. Rev. C 70 (2004) 065804.

24. A. Worley, P.G. Krastev and B.A. Li, Astrophys. J. 685 (2008) 390.

25. C.J. Horowitz and J. Piekarewicz, Phys. Rev. Lett. 86 (2001) 5647; Phys. Rev. C 64 (2001) 062802(R); Phys. Rev. C 66 (2002) 055803.

26. J. Carriere, C.J. Horowitz, and J. Piekarewicz, Astrophys. J. 593 (2003) 463.

27. J. Xu, L.W. Chen, B.A. Li, and H.R. Ma, Phys. Rev. C 79 (2009) 035802; Astrophys. J. 697 (2009) 1549.

28. Thermodynamics, H.B. Callen, Wiley (New York, 1985).

29. C.B. Das, S. Das Gupta, C. Gale, and B.A. Li, Phys. Rev. C 67 (2003) 034611.

30. J. Arponen, Nucl. Phys. A 191 (1972) 257. 